\title{
Downregulation of hTERT contributes to ovarian cancer apoptosis and inhibits proliferation of ovarian cancer cells
}

\author{
Qing-An-Zi Wang, Xiaolei Liang, Yongxiu Yang \\ Department of Obstetrics and Gynecology, the First Hospital of Lanzhou University, Key Laboratory for Gynecologic Oncology Gansu Province, \\ Lanzhou 730000, China \\ Contributions: (I) Conception and design: QAZ Wang, Y Yang; (II) Administrative support: Y Yang; (III) Provision of study materials or patients: QAZ \\ Wang; (IV) Collection and assembly of data: Y Yang; (V) Data analysis and interpretation: X Liang; (VI) Manuscript writing: All authors; (VII) Final \\ approval of manuscript: All authors. \\ Correspondence to: Yongxiu Yang. Department of Obstetrics and Gynecology, the First Hospital of Lanzhou University, No. 1 Donggang West Road, \\ Chengguan District, Gansu Province, Lanzhou 730000, China. Email: yongxiuyang@163.com.
}

Background: Our study aims to study the effects of the exogenous human telomerase reverse transcriptase (hTERT) interfering gene on the ovarian cancer cell line SKOV3 through proliferation and apoptosis.

Methods: Lipofectamine TM2000 was used to transfer the hTERT interfering gene into the SKOV3 cells. After a predetermined amount of time after transfection with the hTERT interfering genes, the expression of the tumor-related genes was detected using real-time quantitative polymerase chain reaction (RTqPCR), and relative protein level was detected using western blot analysis. Cell morphology was acquired by microscopy. Cell viability was detected by middle-time-spray (MTS), and cell cycle and apoptosis were detected by flow cytometry.

Results: Forty-eight hours after transfection, the expression of tumor-related proteins in the experimental group was increased compared with the control group, and the difference was statistically significant $(\mathrm{P}<0.05)$. The cell morphology showed a significant difference between the control group and the hTERT shRNA group. Furthermore, 48 and $72 \mathrm{~h}$ after transfection with the hTERT interfering gene, the cell viability inhibition rates of the hTERT shRNA group were $0.77 \pm 0.02$ and $0.88 \pm 0.01$ respectively. Compared with the control group, the cell viability inhibition rates were $11.97 \pm 2.37$ (\%) and $18.72 \pm 1.01$ (\%), respectively, with statistically significant differences $(\mathrm{P}=0.009, \mathrm{P}=0.004)$. Flow cytometry detected the apoptosis peak of SKOV3 cells in the experimental group $48 \mathrm{~h}$ after transfection with the hTERT interfering gene. Propidium iodide (PI) and Annexin V-FITC revealed that the apoptotic cells accounted for $18.13 \%$ of the total number, which was significantly higher than the $3.85 \%$ demonstrated by the control group.

Conclusions: The amount and activity of SKOV3 cells in ovarian cancer was decreased after the exogenous introduction of the hTERT interfering gene.

Keywords: Ovarian cancer; hTERT gene; downregulation; proliferation; apoptosis

Submitted Aug 06, 2019. Accepted for publication Dec 26, 2019.

doi: $10.21037 /$ tcr.2020.01.39

View this article at: http://dx.doi.org/10.21037/tcr.2020.01.39

\section{Introduction}

Among gynecological malignant tumors, ovarian cancer ranks third in incident rate in the female reproductive system. Also, ovarian cancer has the highest mortality rate among all malignant tumors (1-3). The incidence rate of ovarian cancer has become the third highest of the female reproductive system tumors, and the mortality rate ranks the first among gynecological malignant tumors (1-3). Early diagnosis and treatment options are vital for recovery $(4,5)$; thus, our study focused on ovarian cancer prevention and treatment. 
Finding novel effective drugs with fewer side-effects is the focus of cancer research $(6,7)$. In recent years, many researchers have been devoted to developing new antiovarian cancer drugs in order to find more effective treatments (8). Therefore, it has become necessary to understand the biological mechanisms of ovarian cancer. hTERT is a crucial nutrient factor in primary tumor and cancer cell lines. It has been shown to have a high expression among tumors, while there being no expression in normal tissues. Therefore, the clinical values and multiple biological effects are not understood clearly $(9,10)$.

Recently, several genes have been reported to exert apoptosis-inducing activity for human cancers. In our study, we investigated and compared the apoptosis-inducing activity of p21, p53, cmc-y, tumor necrosis factor (TNF)related apoptosis-inducing ligand (TRAIL) expression in SKOV3 cell lines (11). The hTERT gene was silenced in this study after electrotransfection into ovarian cancer SKOV3 cells to investigate the therapeutic effect of this method on ovarian cancer persistence.

\section{Methods}

\section{Materials and reagents}

The human ovarian cancer cell line SKOV3 was bought from the ATCC corporation (USA). Vector RNAi-Ready pSIREN-DNR-dsred-express was bought from Santa Cruz Biotechnology company (USA). Lipofectamine 2000 was obtained from Invitrogen, and McCoy's 5A (modified) cell medium was purchased from Thermo Fisher Scientific (USA). Streptomycin and potassium phosphate buffer (PBS) were also purchased from Hyclone. Meanwhile, the cell lysates were bought from Life Technologies, and Super Signal West Pico chemiluminescent substrate kit was bought from Thermo Scientific. A BCA protein concentration kit (Shanghai Biyuntian biotechnology) and a Alexa Fluor 88 annexinV cell cycle apoptosis kit were also purchased (Invitrogen, USA); all the other reagents were analytical pure (8).

\section{Study methods \\ siRNA design}

shRNA was designed to select RNAi target sites (hTERT mRNA sites 1812-1832 nucleotide, long 19 nt, target sequence: AGCATTGGAATCAGACAGC). In combination with the specification of RNAi ready
Psc-36641-SH-hTERT-expressing plasmid from BD Biosciences Clontech, an shRNA online design tool was used to design oligonucleotide chains that could transcriptionally target hTERT shRNA, including BamH 1 and EcoR 1 restriction sites at both ends and reverse complementary target sequences, which were separated by loop intervals of 9 bp non-homologous sequence (TTCAAGAGA). 3' end with a TTTTTT termination sequence, chemical synthesis Invitrogen company Shanghai branch is from the United States. The two oligonucleotides chain are Sense: 5'-GAT CCAGCATTGGAATCAGACAGCTTCAAGAGAGCT; GTCTGATTCCAATGCTTTTTTTGGAAG - 3 ' and Antisense: 5'- AATTCCTTCCAAAAAAAGCATTGGAA TCAGACAGCTCTCTTGAAGCTGTCTGATTCCA ATGCT -3'. Meanwhile, negative control sequences were designed by the same method.

\section{Cells transfection}

We divided ovarian cancer SKOV3 cells into 4 test groups: the non-transfection group as the control group, the pIRSC2-blank as the blank control group, the transfection (blank group) as the control, the non-specific siRNA vector (pIRSC2-NC) transfection as the negative control (NC group), and the pIRSC2-shTERT transfection as the observation group (shTERT group). According to the procedure provided in the transfection kit, transfection was carried out instantaneously. In the transfection process, $3 \mathrm{~g}$ recombinant plasmid and $6 \mathrm{~mL}$ liposomes were dissolved in $500 \mathrm{~mL}$ serum-free medium to make the transfer solution, and the transfection was suspended after $6 \mathrm{~h}$ with the addition of $2 \mathrm{~mL}$ per well to the final volume of serumfree medium. It was observed that the red fluorescence could be seen in SKOV 3 cells $12 \mathrm{~h}$ after the staining, and the fluorescence expression was strongest at 48-72 h, with the fluorescence still being until day 7 . In this study, the 24-72 h timeframe after transfection was selected as the experimental time. The Psc-36641-SH-hTERT transfection group (SKOV3/p-shRNA) and the blank control group were then established $(12,13)$.

\section{Real-time quantitative polymerase chain reaction (RT-} qPCR)

RT-qPCR detected the expression of hTERT, C-MYC, P21, P53, TRAIL mRNA. GAPDH was used as the control. Total RNA was isolated from SKOV3 cells and was quantified by nucleic acid electrophoresis. When the ratio of $28 \mathrm{~s}$ rRNA to $18 \mathrm{~s}$ rRNA was greater than or equal to 1 , the quality of total RNA extracted was 
qualified. When the expression of all samples all went through GAPDH level correction, according to the $2^{-\Delta \Delta C t}$ formula, the relative expression level of samples was calculated. hTERT primer sequence in upstream was 5'-GGCCTTCACCACCAGCGTGC-3', and in downstream was 5'-AGGACCCCTGCCCAACGGGC-3', with amplification fragment $427 \mathrm{bp}$; within the referring to the sequence of GAPDH primers, upstream was 5 ' - AGGTAGGACGAGGTCGGAGTC-3 ', and downstream was 5'-GAATTAGGTACTAGGATTTC-3' $(14,15)$.

\section{Western blotting}

SKOV3 cell lines were transfected with a plasmid for $48 \mathrm{~h}$. After that, the cells of each group were collected, and proteins were extracted for later analysis. Next, RIPA cell lysate was added to each well of the 6-well plate (PMSF, Protease inhibitor) to extract protein, using the BCA protein concentration determination kit to determine protein concentration. Then, $20 \mathrm{mg}$ protein was transferred to sds-page gel, followed by transfer to PVDF. They were incubated by primary antibody and secondary antibody, and ECL exposure was used to acquire the image and analysis.

\section{Cell proliferation assay}

MTS cell proliferation confirmed SKOV3 cell lines at $36 \mathrm{~h}$ after transfection. After inoculation, the cells were cultured in a 96-well culture plate, and the number of cells per well was the same. The proliferation of MTS cells was detected after the incubators were set for $12 \mathrm{~h}$. The proliferation of SKOV3 cells in each well was detected, continuously for 3 days by MTS assay kit. MTS was added to each well and mixed with diluent $100 \mu \mathrm{L}(10 \mu \mathrm{L}$ MTS reagent and $90 \mathrm{~mL} \mathrm{10 \%} \mathrm{FBS+1640} \mathrm{medium)} \mathrm{in} \mathrm{a} \mathrm{cell} \mathrm{culture} \mathrm{plate} \mathrm{at}$ $37{ }^{\circ} \mathrm{C}$ and incubated for $2 \mathrm{~h}$ in the dark. Finally, the absorbance value at $490 \mathrm{~nm}$ was detected by a microplate reader. The conditions of each test were consistent (12).

\section{Flow cytometry detection}

Apoptotic cells were harvested and transfected by flow cytometry, centrifuged at $1,000 \mathrm{r} / \mathrm{min}$, precooled, fixed with $75 \%$ ethanol, and stained with propidium iodide (PI). Meanwhile, apoptotic cells were labeled with fitc-annexin $\mathrm{v}$, and the apoptotic effect of SKOV3 cells was detected by flow cytometry. The specific steps were conducted in strict accordance with the instructions of the kit, and the optical density value of the test strip was measured by image analysis technology. The test operation of the SKOV3 group was repeated 3 times (16).

\section{Statistical processing analysis}

SPSS version 19.0 was used as the statistical analysis software while analysis and measurement data were conducted by t-test. Data are expressed as the mean \pm SD (standard deviation) of at least 3 separate experiments performed in triplicate. $\mathrm{P}$ values $<0.05$ were considered statistically significant.

\section{Results}

\section{The effect of bTERT on the expression of cell cycle-and apoptosis-related proteins}

To determine whether hTERT contributes to the proliferation of SKOV3 cells, we checked the hTERT expression level in SKOV3 cell lines and found that hTERT siRNA knockdown was performed in SKOV3 cell lines. The RT-PCR results showed that p21, p53, and TRAIL expression was significantly increased in the SKOV3 cells $36 \mathrm{~h}$ after transfection with $2 \mathrm{mmol} / \mathrm{L}$ hTERT shRNA sequence. In contrast, the cell cycle gene (c-myc) expression was significantly decreased $36 \mathrm{~h}$ after transfection with $2 \mathrm{mmol} / \mathrm{L}$ hTERT shRNA sequence. This indicates that knockdown hTERT could upregulate tumor related genes (p21, p53, and TRAIL) and inhibit c-myc. The western blot analysis showed the same trend with RT-PCR (Figure 1).

\section{The effect of inbibition of bTERT on the proliferation of SKOV 3 cells}

SKOV3 cells transfected with hTERT shRNA showed significantly slower growth and reduced proliferation compared with SKOV3 cells transfected with empty plasmid on the second and third days after transfection (Figure 2).

\section{The effect of bTERT on the morphology of SKOV3 cells and the apoptosis-inducing effect of HTERT on SKOV3 cells}

SKOV3 cells were transfected with hTERT ( $2 \mathrm{mmol} / \mathrm{L})$ for $48 \mathrm{~h}$, and the morphological changes induced by the hTERT treatments were examined under an inverted microscope. The NC group cell structure was clear, and the cell nucleus was full. Compared with the NC group, the number of the SKOV3 cells was markedly reduced after treatment with $2 \mathrm{mmol} / \mathrm{L}$ hTERT for $48 \mathrm{~h}$. The 
A TERT mRNA

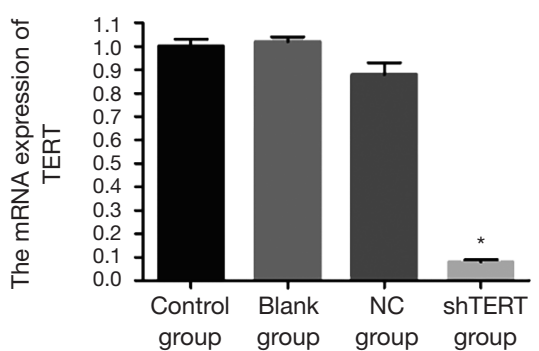

p21 mRNA

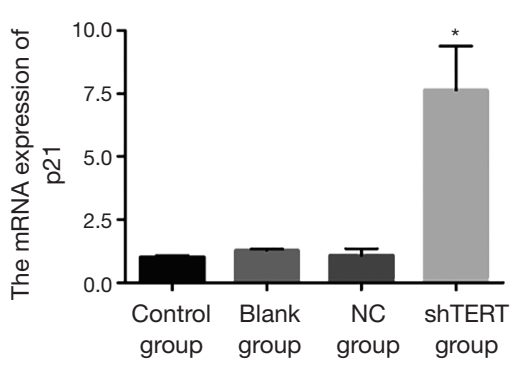

TRAIL mRNA

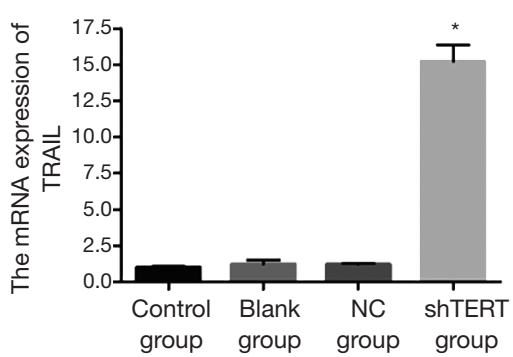

B
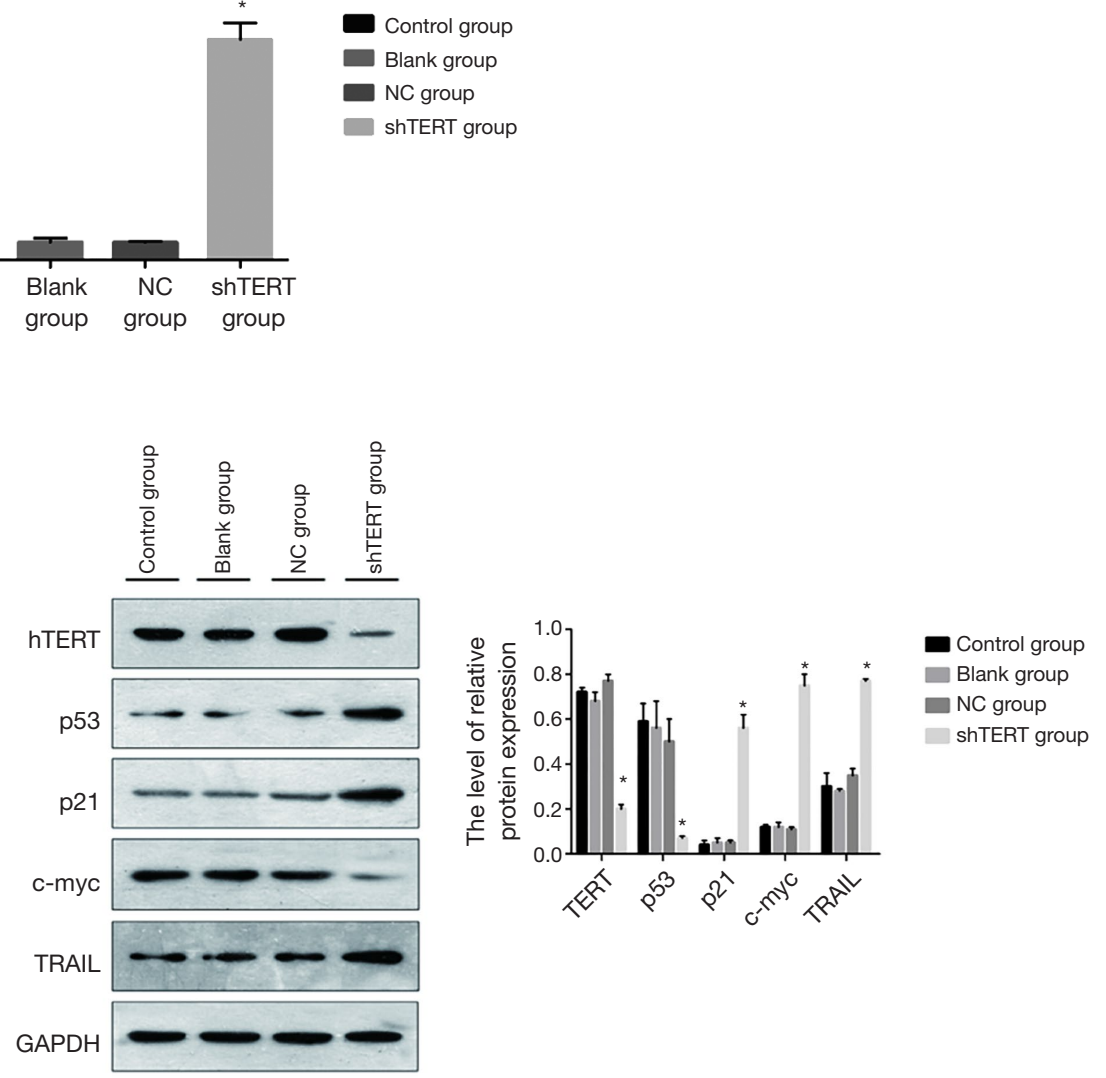

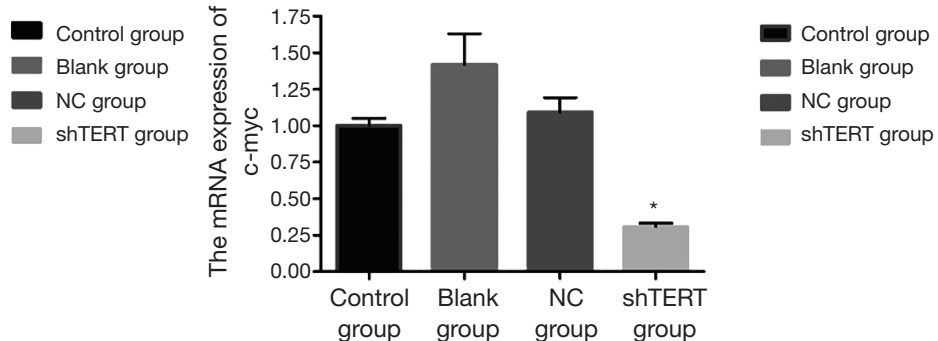

Control group

Blank group

NC group

shTERT group

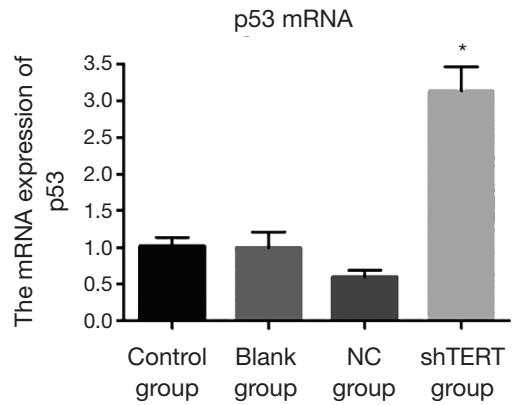

Control group

Blank group

NC group

shTERT group

Figure 1 The expression of hTERT mRNA, p53, p21, c-myc, and TRAIL was detected by RT-PCR (A) and western blot (B). *P<0.05 vs. NC group. 
A
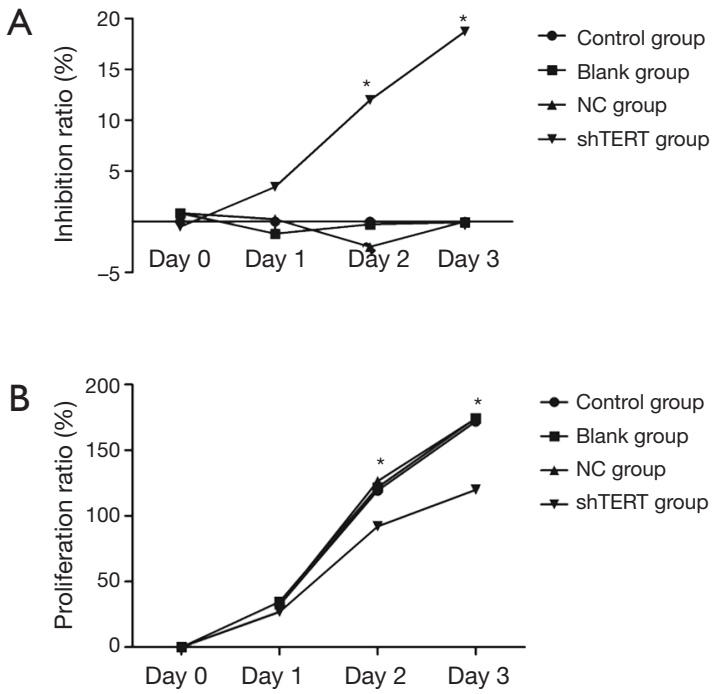

Figure 2 Proliferation and inhibitory effect of hTERT on SKOV3 cells detected by MTS assay. SKOV3 cells were transfected with hTERT for 1 day, 2 days, and 3 days. (A) Inhibition rate $=(1-$ od value mean/control group OD value mean) $\times 100 \%$ (at the same time), (B) proliferation rate $=(\mathrm{OD}$ value mean at other time points $/ 0 \mathrm{~h}$ OD value mean -1$) \times 100 \%$ (at the same time). ${ }^{*} \mathrm{P}<0.05$ compared with the corresponding $\mathrm{NC}$ group.
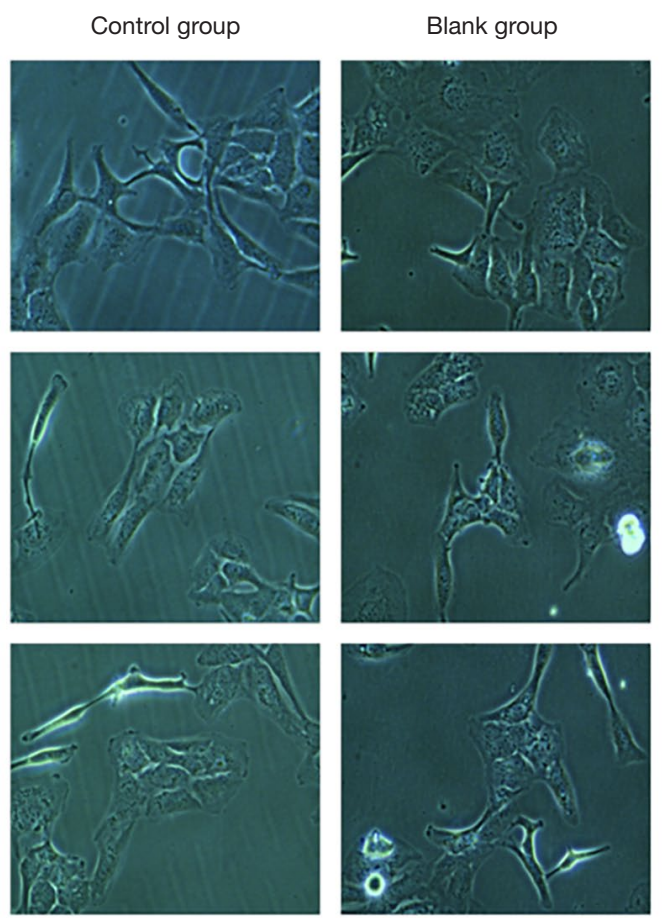

hTERT-treated cells became long and thin and displayed an elongated spindle-shaped morphology. Some hTERTtreated cells even appeared star-shaped (Figure 3).

\section{The effect of inbibition of hTERT on the apoptosis rate of SKOV 3 cells}

The flow cytometric analysis results showed that the SKOV3 cells underwent apparent apoptosis after treatment with hTERT shRNA. The number of early apoptotic cells and late apoptotic cells both increased after treatment with hTERT shRNA compared with the control group (Figure 4). The total apoptosis rate in the hTERT shRNA group was $18.66 \pm 1.33$, which was significantly higher than that in the control group $(2.92 \pm 0.33)$, blank group (3.02 \pm 0.65$)$, and $\mathrm{NC}$ group $(3.66 \pm 0.34)$.

\section{Discussion}

In clinical practice, radiation and chemotherapy can not be tolerated by normal tissues, which in turn affects the survival rate for ovarian cancer patients. Currently, gene therapy is a hot-topic issue in the domestic and overseas fields of biological research. New genes have been discovered as one

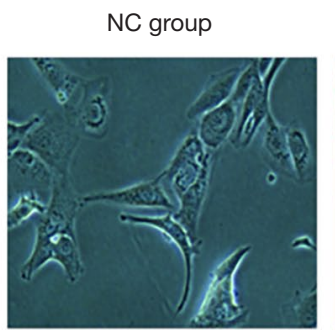

shTERT group
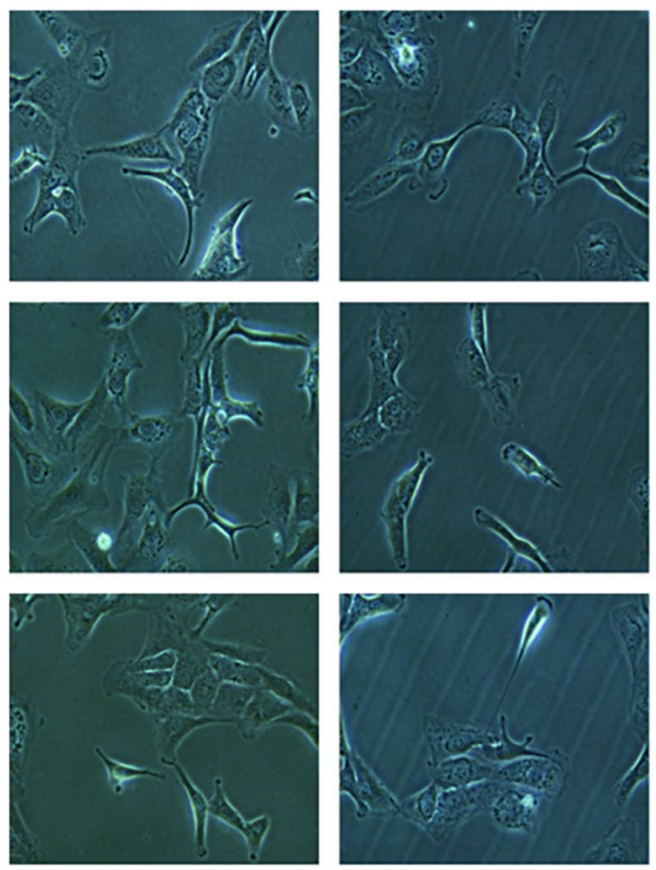

Figure 3 The morphology changes of SKOV3 cells treated with hTERT shRNA for $48 \mathrm{~h}(10 \times 40)$. 
A
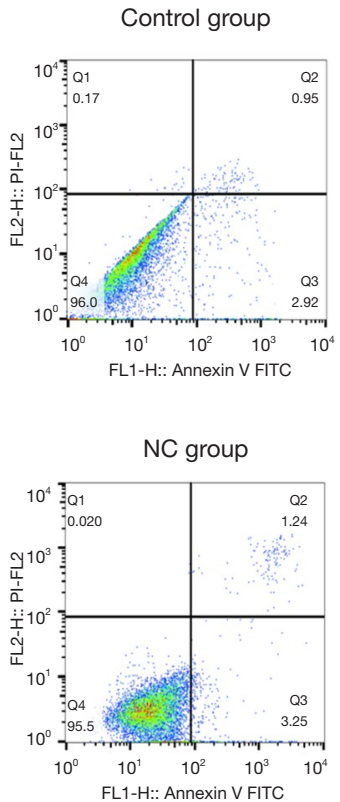

Blank group
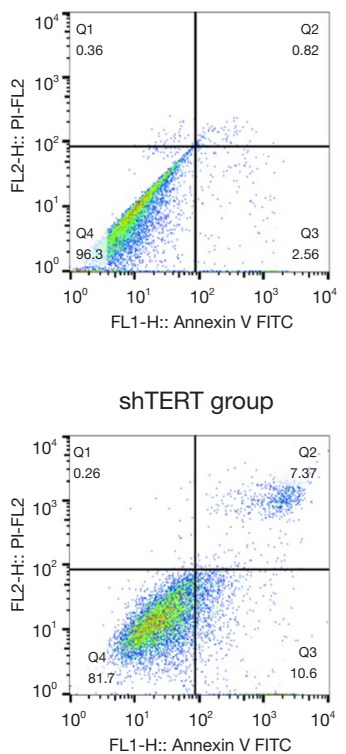

B

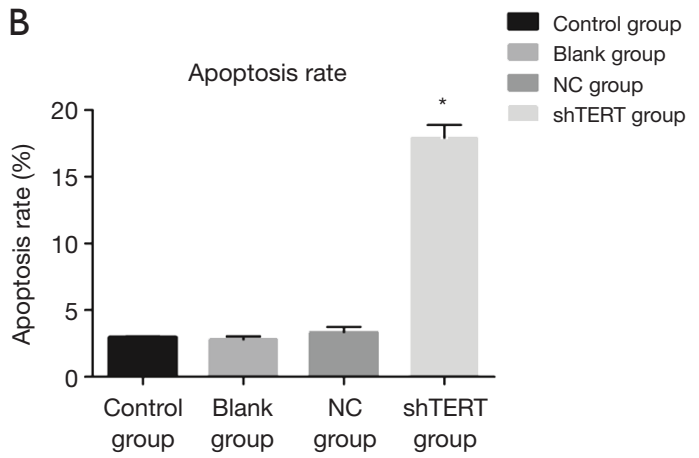

Figure 4 Apoptosis-induced effects of hTERT on SKOV3 cells by flow cytometry. (A) Apoptosis rates of SKOV3 cells treated with hTERT at $2 \mathrm{mmol} / \mathrm{L}$ for $48 \mathrm{~h}$; (B) the column diagram of apoptosis rates of SKOV3 cells treated with hTERT at $2 \mathrm{mmol} / \mathrm{L}$ for $48 \mathrm{~h}$. ${ }^{*}<<0.05 v s$. NC group.

of the treatment methods for ovarian cancer, opening new possibilities in treatment $(17,18)$.

In recent years, the development of RNAi as a novel silencing gene technology has allowed for the determination of the genetic signature of siRNA. Our study established that the expression of hTERT siRNA, eukaryotic expression plasmid, and negative control plasmid were able to be successfully transfected into the ovarian cancer SKOV3 cell line, with a transfection efficiency of more than $50 \%$, indicating that the transfection was successful.

As a result, the expression of hTERT mRNA was significantly decreased. DNA was cloned into a plasmid and reconstituted into hTERT mRNA-siRNA. The expression vector of hTERT mRNA was detected by RT-pcr assay, indicating target gene silencing, which is consistent with the research results of Chen et al. (11). Research data showed that telomerase is a reverse transcriptase, and can maintain telomere length.

hTERT is a catalytic subunit of telomerase and can be reverse transcribed using RNA as a template to synthesize telomerase. hTERT is closely related to cell growth, development, proliferation, and differentiation. hTERT can significantly inhibit the growth of carcinoma cells, which exist in multiple organisms. hTERT expression is inhibited in normal tissues, but not in cancer cells $(15,19)$.

However, there was high expression of hTERT that was found in tumor lines. It was also found that the hTERT gene could be transfected by electrotransfection to achieve an effective and lasting effect. Additionally, it was found that electroporation could be used for transfection because the target gene transient expression rate is much higher than the liposome transfection method so that the electro transfer can be an effective and simple method. This study showed that siRNA targeted silencing hTERT gene could be electro transferred into ovarian cancer SKOV3 cells, which can play an active role in guiding the treatment of ovarian cancer. hTERT genes may supply novel targets for gene therapy of ovarian cancer and other tumors.

\section{Acknowledgments}

Funding: None.

\section{Footnote}

Conflicts of Interest: All authors have completed the ICMJE uniform disclosure form (available at http://dx.doi. org/10.21037/tcr.2020.01.39). The authors have no conflicts 
of interest to declare.

Ethical Statement: The authors are accountable for all aspects of the work in ensuring that questions related to the accuracy or integrity of any part of the work are appropriately investigated and resolved. The study was conducted in accordance with the Declaration of Helsinki (as revised in 2013). Institutional ethical approval and informed consent were waived.

Open Access Statement: This is an Open Access article distributed in accordance with the Creative Commons Attribution-NonCommercial-NoDerivs 4.0 International License (CC BY-NC-ND 4.0), which permits the noncommercial replication and distribution of the article with the strict proviso that no changes or edits are made and the original work is properly cited (including links to both the formal publication through the relevant DOI and the license). See: https://creativecommons.org/licenses/by-nc-nd/4.0/.

\section{References}

1. Briggs MT, Condina MR, Ho YY, et al. MALDI Mass Spectrometry Imaging of Early- and Late-Stage Serous Ovarian Cancer Tissue Reveals Stage-Specific N-Glycans. Proteomics 2019;19:e1800482.

2. Chelariu-Raicu A, Coleman RL, Sood AK. Anti-Angiogenesis Therapy in Ovarian Cancer: Which Patient is It Most Likely to Benefit? Oncology (Williston Park) 2019;33.

3. Lohse I, Azzam DJ, Al-Ali H, et al. Ovarian Cancer Treatment Stratification Using Ex Vivo Drug Sensitivity Testing. Anticancer Res 2019;39:4023-30.

4. Aghamiri S, Mehrjardi KF, Shabani S, et al. NanoparticlesiRNA: a potential strategy for ovarian cancer therapy? Nanomedicine (Lond) 2019;14:2083-100.

5. Herrera FG, Irving M, Kandalaft LE, et al. Rational combinations of immunotherapy with radiotherapy in ovarian cancer. Lancet Oncol 2019;20:e417-33.

6. Bernstein-Molho R, Barnes-Kedar I, Ludman MD, et al. The yield of full BRCA1/2 genotyping in Israeli Arab high-risk breast/ovarian cancer patients. Breast Cancer Res Treat 2019;178:231-7.

7. Korach J, Colombo N, Mendiola C, et al. Outcome according to residual disease (surgeon's report vs prechemotherapy imaging) in patients with bevacizumabtreated ovarian cancer: Analysis of the ROSiA study. J Surg Oncol 2019;120:786-93.

8. Kang M, Xia P, Hou T, et al. MicroRNA-190b inhibits tumor cell proliferation and induces apoptosis by regulating Bcl-2 in U2OS osteosarcoma cells. Pharmazie 2017;72:279-82.

9. Bettegowda C, Yip S, Jiang B, et al. Prognostic significance of hTERT (human telomerase reverse transcriptase) promoter region mutations C228T and C250T for overall survival in spinal chordomas. Neuro Oncol 2019. [Epub ahead of print].

10. Ho ST, Jin R, Cheung DH, et al. The PinX1/NPM interaction associates with hTERT in early-S phase and facilitates telomerase activation. Cell Biosci 2019;9:47.

11. Chen D, Chen L, Xiao L. The progress study of tumor suppressor gene and apoptosis gene in nasal polyps. Lin Chung Er Bi Yan Hou Tou Jing Wai Ke Za Zhi 2015;29:2099-102.

12. Wang W, Yang J, Yu F, et al. MicroRNA-122-3p inhibits tumor cell proliferation and induces apoptosis by targeting Forkhead box O in A549 cells. Oncol Lett 2018;15:2695-9.

13. Wu L, Zhang X, Lin X, et al. Inhibition of X-linked inhibitor of apoptosis protein enhances anti-tumor potency of pure total flavonoids on the growth of leukemic cells. Exp Ther Med 2018;15:2020-6.

14. Pei ZJ, Zhang ZG, Hu AX, et al. miR-122-5p inhibits tumor cell proliferation and induces apoptosis by targeting MYC in gastric cancer cells. Pharmazie 2017;72:344-7.

15. Shepelev MV, Kalinichenko SV, Saakian EK, et al. Xenobiotic Response Elements (XREs) from Human CYP1A1 Gene Enhance the hTERT Promoter Activity. Dokl Biochem Biophys 2019;485:150-2.

16. Li P, Zhang Y, Pan H, et al. Doxorubicin-loaded pHsensitive dextran-retinal nanoparticles suppress tumor growth by inducing both apoptosis and cell senescence. J Control Release 2015;213:e88-9.

17. Sanada Y, Harada M, Kunitomi C, et al. A Japanese nationwide survey on the cryopreservation of embryos, oocytes and ovarian tissue for cancer patients. J Obstet Gynaecol Res 2019;45:2021-8.

18. Zhou X, Liu Y, Liu Q, et al. Point-of-care Ratiometric Fluorescence Imaging of Tissue for the Diagnosis of Ovarian Cancer. Theranostics 2019;9:4597-607.

19. Ludlow AT, Slusher AL, Sayed ME. Insights into Telomerase/hTERT Alternative Splicing Regulation Using Bioinformatics and Network Analysis in Cancer. Cancers (Basel) 2019;11. doi: 10.3390/cancers11050666.

Cite this article as: Wang QAZ, Liang X, Yang Y. Downregulation of hTERT contributes to ovarian cancer apoptosis and inhibits proliferation of ovarian cancer cells. Transl Cancer Res 2020;9(3):1448-1454. doi: 10.21037/ tcr.2020.01.39 